\title{
Divertículo del seno sigmoide: Posible causa de acúfeno pulsátil
}

\section{Diverticulum of the Sigmoid Sinus: Possible Cause of Pulsatile Tinnitus}

\author{
Carlos García-Recio ${ }^{1 \odot}$ Jaime Iglesias-Gordo ${ }^{2}$ Daniella Carolina Laguado-Bulgheroni ${ }^{1}$
}

${ }^{1}$ Servicio de Otorrinolaringología, Complejo Hospitalario de Cáceres,
Cáceres, España
${ }^{2}$ OSATEK-Donostia, Donostia, Gipuzkoa, España

Rev Argent Radiol 2019;83:130-132.

Estimados Editores,

Las anomalías del seno sigmoide son una posible causa de acúfeno pulsátil. Ese tipo de acúfeno o tinnitus se caracteriza por mantener un ritmo pulsátil sincronizado con el ritmo cardíaco, sin estímulo acústico que lo genere. Las estructuras vasculares están a menudo implicadas en la producción de la sintomatología, en las cuales un curso anormal o aberrante de un vaso, una dehiscencia o falta de normal cobertura ósea o bien una estenosis pueden ocasionar flujo turbulento generando vibraciones que se pueden transmitir al sistema auditivo. ${ }^{1}$ Algunos ejemplos son la arteria carótida interna aberrante, dehiscente o con estenosis, posición alta o dehiscencia del bulbo de la vena yugular, divertículo o dehiscencia del seno venoso sigmoide, fístulas arteriovenosas, persistencia de arteria estapedial o tumores.

Presentamos el caso de una paciente de 75 años que consulta por hipoacusia y molestias óticas inespecíficas en el oído derecho. En la exploración el oído derecho era normal, pero en el izquierdo se visualizaba una masa azulada, pulsátil y depresible en la pared posterior del CAE óseo, que se movilizaba con la compresión cervical. La audiometría presentaba una hipoacusia neurosensorial compatible con presbiacusia. Se inició el estudio de la lesión mediante tomografía computarizada (TC) (- Figuras 11 y 2 ), visualizándose en las imágenes con ventana ósea un voluminoso defecto en la pared del hueso temporal adyacente al seno sigmoide izquierdo (placa sigmoide), ocupado por una masa con densidad de tejidos blandos que erosiona la pared ósea, celdillas, antro y hueso en toda

(1)García-Recio, Carlos's ORCID is https://orcid.org/0000-00021274-7713.

received

October 28, 2018

accepted

May 6, 2019
Address for correspondence Carlos García Recio, MD, Servicio de Otorrinolaringología, Complejo Hospitalario de Cáceres, Avenida de los Pilares, $n^{\circ} 3,1^{\text {a }} \mathrm{A}, 10002$ Cáceres, España, (e-mail: carlosgarciarecio.cgr@gmail.com). su extensión hasta alcanzar el conducto auditivo externo. También hay aumento de neumatización de celdillas mastoideas respecto al lado contralateral.

Dados los hallazgos, se decidió ampliar estudio mediante resonancia magnética (RM) y angio-RM (-Figuras 3 y 4), apreciando que el defecto óseo visualizado en la TC era ocasionado por un divertículo gigante del seno sigmoide izquierdo. Con ese diagnóstico se planteó actuación terapéutica pero, teniendo en cuenta que el divertículo era asintomático y que la paciente tenía numerosas comorbilidades, se decidió observación clínica y radiológica informando del importante riesgo de sangrado, haciendo necesaria la vigilancia estrecha por si fuera preciso tratamiento.

El divertículo del seno sigmoide (DSS), es un saco bien circunscrito donde el seno sigmoide sobresale focalmente hacia el área mastoidea a través de la pared dehiscente del seno. ${ }^{2}$ Además del divertículo, existen otras anomalías del mismo como el aneurisma del seno sigmoide, siendo un término intercambiable con el anterior, al considerarse ambas malformaciones del seno sigmoide. Otra alteración es la dehiscencia del seno sigmoide (DhSS), que es un defecto en la continuidad de la cobertura ósea del seno que permite la exposición del seno a las celdas mastoideas.

La existencia de diferentes definiciones para las anomalías del seno sigmoide, la ausencia de directrices establecidas para su diagnóstico y una descripción relativamente reciente hacen pensar que su prevalencia pueda estar infraestimada, pudiendo llegar a ser la primera causa de acúfeno pulsátil, con una prevalencia de hasta el $23 \%$ de DSS en pacientes con ese tipo de acúfeno. ${ }^{3}$

El DSS se suele localizar en la pared externa de la curva superior o en el segmento descendente del seno sigmoide,
Copyright @ 2019, Sociedad Argentina de Radiología. Publicado por Thieme Revinter Publicações Ltda., Rio de Janeiro, Brazil. Todos los derechos reservados.
DOI https://doi.org/

10.1055/s-0039-1692151. ISSN 1852-9992.

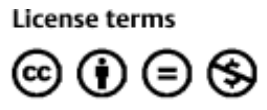




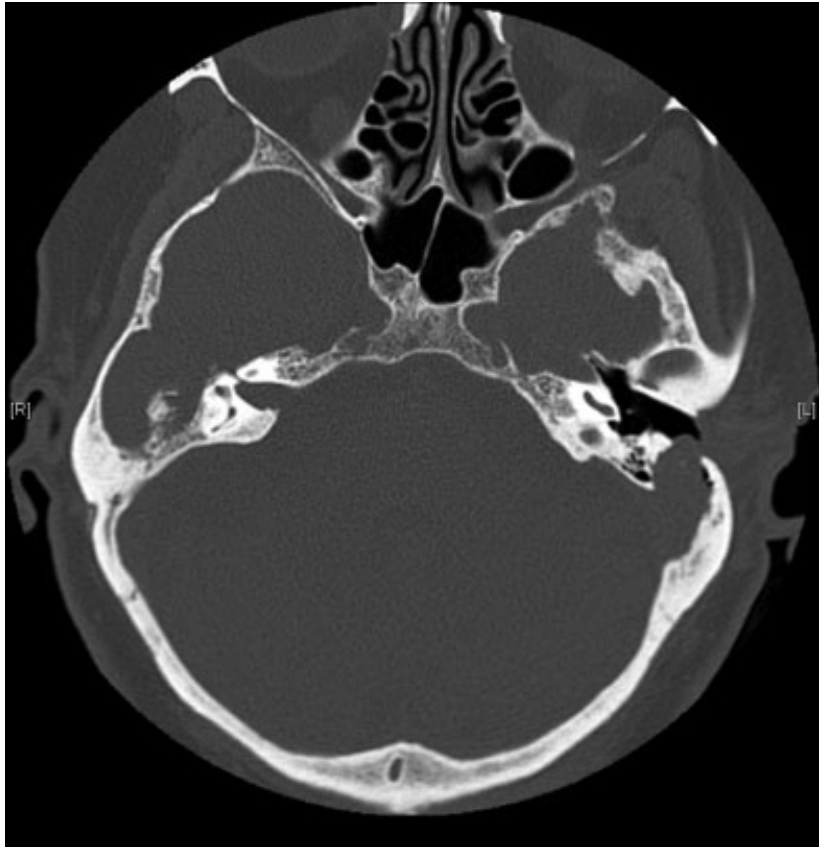

Fig. 1 TC axial con ventana ósea donde se aprecia un voluminoso defecto en la pared del hueso temporal adyacente al seno venoso sigmoide izquierdo ocupado por una masa con densidad de tejidos blandos que erosiona el antro, las celdillas y la pared externa temporal hasta alcanzar el conducto auditivo externo. Hay aumento de la neumatización de las celdillas mastoideas respecto al lado contralateral.

asociándose frecuentemente a la dehiscencia de la placa sigmoide del hueso temporal, con aumento de la neumatización de las celdillas mastoideas. ${ }^{4}$

Las teorías más extendidas sobre la formación del DSS son fundamentalmente dos: una que asocia su formación a la existencia de hipertensión intracraneal concomitante que podría incrementar las fuerzas de impacto sobre el hueso, erosionándolo y favoreciendo la aparición del DSS, la DhSS o ambos. ${ }^{5}$ La segunda sugiere como causa la estenosis del seno transverso (que también podría asociarse a la presencia de hipertensión intracraneal), la cual generaría un aumento en las velocidades de flujo en la zona proximal a la estenosis o bien aumento del volumen en el seno dominante si hay estenosis contralateral, con erosión progresiva de la pared ósea del seno venoso y

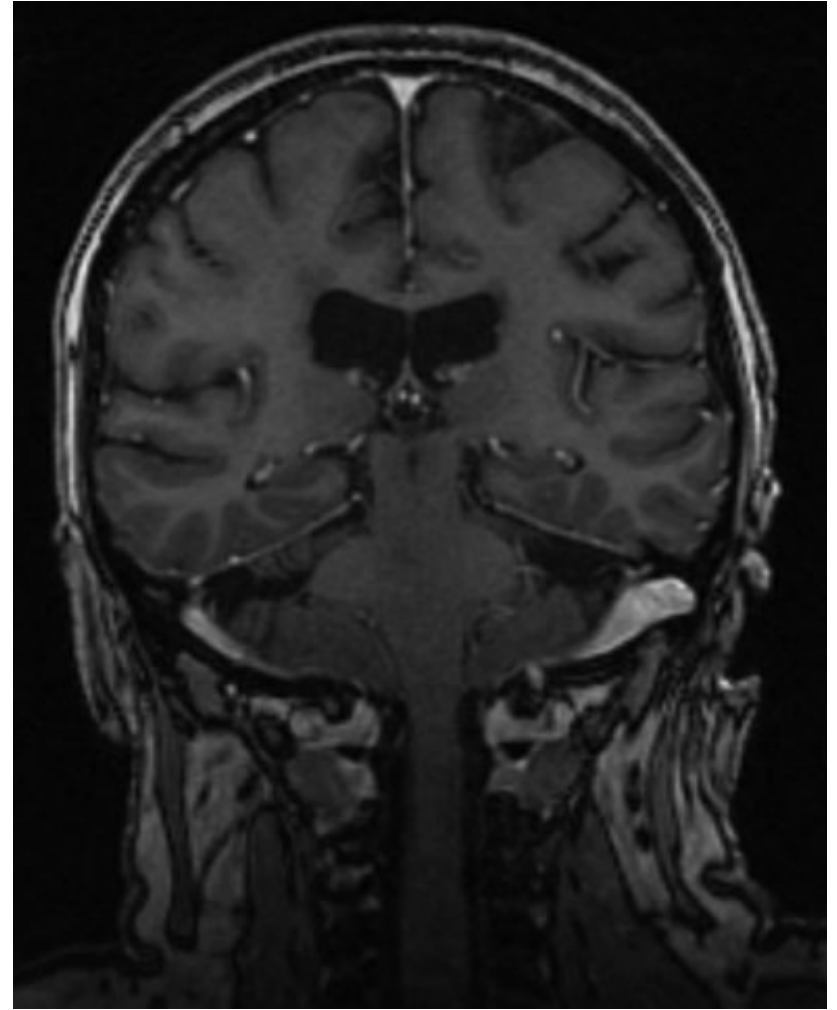

Fig. 3 Imagen coronal de resonancia magnética eco de gradiente ponderada en T1 con contraste paramagnético. Se aprecia la expansión del seno sigmoideo izquierdo en forma de divertículo gigante a través del hueso temporal izquierdo.

favoreciendo la DhSS con ensanchamiento progresivo y formación del DSS. ${ }^{6}$

En cuanto al diagnóstico, la TC es la imagen de elección para valorar posibles anomalías de la pared del seno sigmoide. $^{7}$ La angio-TC realizada a los 20 segundos tras la visualización de contraste en las arterias carótidas comunes permitiría evaluar tanto las posibles causas arteriales como venosas del acúfeno pulsátil. Las imágenes deben reconstruirse con algoritmos de alta resolución en los planos axial y coronal, valorando en profundidad las imágenes con ventana ósea. La RM sin y con contraste, incluyendo la venografía por RM, sería una prueba

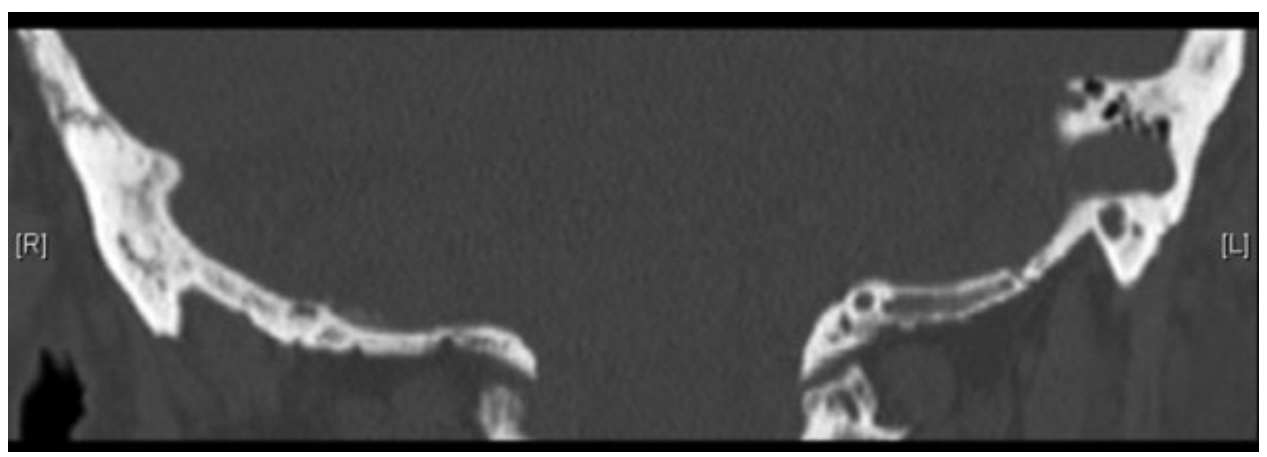

Fig. 2 TC coronal con ventana ósea donde se aprecia el voluminoso defecto en la pared ósea temporal del seno sigmoide izquierdo, ocupado por una masa con densidad de tejidos blandos y con aumento de la neumatización de celdillas mastoideas. 


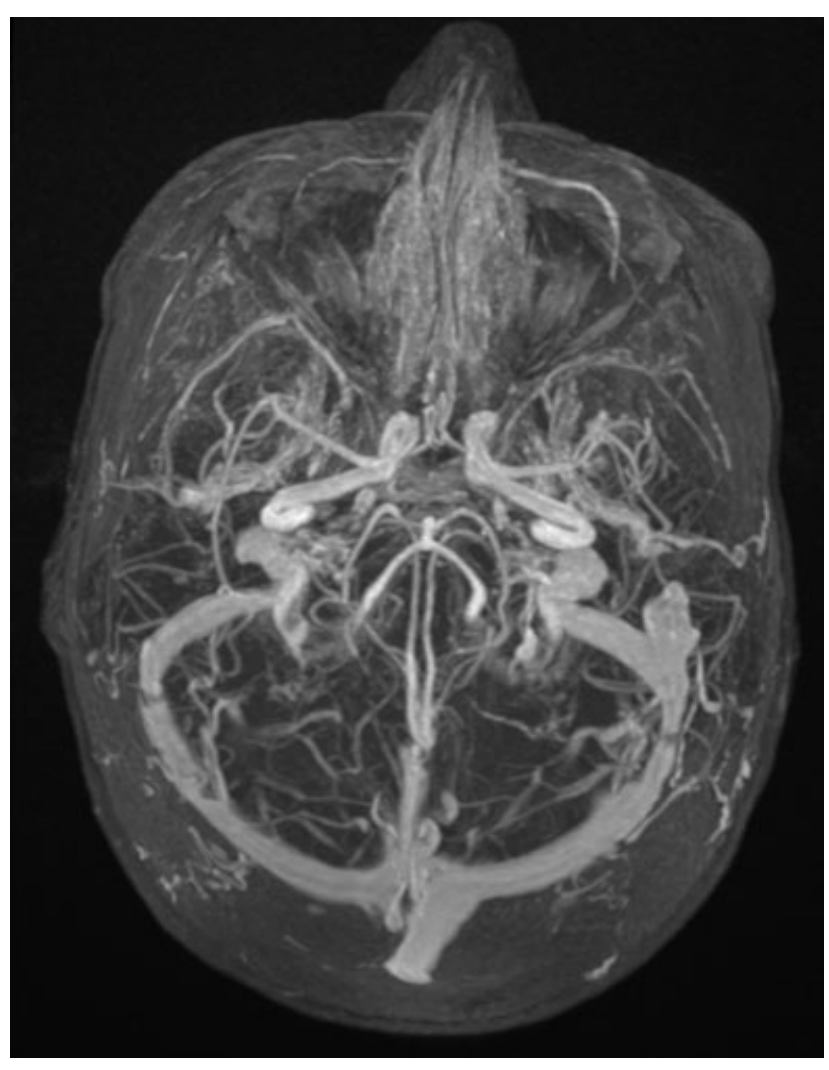

Fig. 4 Imagen axial de angio-RM eco de gradiente 3DTOF (tiempo de vuelo o time of flight en inglés), en la que se aprecia claramente el voluminoso divertículo procedente del seno sigmoide izquierdo.

complementaria a la TC, pudiendo ser útil para el diagnóstico y el seguimiento.

Continúa en discusión cuál es el manejo terapéutico más adecuado, existiendo las posibilidades del tratamiento endovascular y quirúrgico para tratar de solventar la sintomatología derivada de las anomalías del seno sigmoide, sin llegar a un claro consenso en cuál de las dos opciones ofrece mayores garantías. Liu y col., ${ }^{4}$ sí establece un menor riesgo de complicaciones en los pacientes tratados quirúrgicamente, estableciendo en su serie un mayor riesgo de embolismo, además de precisar un tratamiento antiagregante prolongado posterior en los pacientes a los que se sometió a tratamiento endovascular. ${ }^{8-10}$ En lo que sí parece haber consenso es en tratar únicamente a los pacientes sintomáticos, estableciendo un manejo conservador $\mathrm{y}$ expectante en los pacientes asintomáticos.

Conflictos de Intereses

Ninguno de nosotros tenemos conflictos de intereses.

\section{Bibliografía}

1 Branstetter BF IV, Weissman JL. The radiologic evaluation of tinnitus. Eur Radiol 2006;16(12):2792-2802

2 Wang GP, Zeng R, Liu ZH, et al. Clinical characteristics of pulsatile tinnitus caused by sigmoid sinus diverticulum and wall dehiscence: a study of 54 patients. Acta Otolaryngol 2014;134(01):7-13

3 Schoeff S, Nicholas B, Mukherjee S, Kesser BW. Imaging prevalence of sigmoid sinus dehiscence among patients with and without pulsatile tinnitus. Otolaryngol Head Neck Surg 2014;150(05):841-846

4 Liu Z, Chen C, Wang Z, et al. Sigmoid sinus diverticulum and pulsatile tinnitus: analysis of CT scans from 15 cases. Acta Radiol 2013;54(07):812-816

5 Liu Z, Dong C, Wang X, et al. Association between idiopathic intracranial hypertension and sigmoid sinus dehiscence/ diverticulum with pulsatile tinnitus: a retrospective imaging study. Neuroradiology 2015;57(07):747-753

6 Eisenman DJ, Raghavan P, Hertzano R, Morales R. Evaluation and treatment of pulsatile tinnitus associated with sigmoid sinus wall anomalies. Laryngoscope 2018;128(Suppl 2):S1-S13; Epub ahead of print

7 Reardon MA, Raghavan P. Venous Abnormalities Leading to Tinnitus: Imaging Evaluation. Neuroimaging Clin N Am 2016;26 (02):237-245

8 Paramasivam S, Furtado S, Shigamatsu T, Smouha E. Endovascular Management of Sigmoid Sinus Diverticulum. Intervent Neurol 2016;5(1-2):76-80

9 Cuellar H, Maiti T, Patra DP, Savardekar A, Sun H, Nanda A. Endovascular Treatment of Pulsatile Tinnitus by Sigmoid Sinus Aneurysm: Technical Note and Review of the Literature. World Neurosurg 2018;113:238-243

10 Yeo WX, Xu SH, Tan TY, Low YM, Yuen HW. Surgical management of pulsatile tinnitus secondary to jugular bulb or sigmoid sinus diverticulum with review of literature. Am J Otolaryngol 2018;39 (02):247-252 